\title{
Realtime Covid19 Tracker Using React
}

\author{
Kabir Narang, Ms. Shivangi Kaushal, Ms. Ashima Gambhir
}

\begin{abstract}
Analysing the data of a deadly pandemic that has created a mess in this wonderful world and caused a lot of deaths is a need of this hour such that we can easily take preventive measures and hold this pandemic growth and eradicate it with certain measures and proper planning and a study is needed to analyse whether any inter-mediate hosts have facilitated the transmission of the virus to humans or vice versa and this could only be done if precise data is analysed [1][6]. The main objective behind writing this paper is to present an idea that how knowledge to an emerging frontend technology like React JS can lead to a wonderful user interface that can serve as a data analyser web software for this deadly pandemic. The paper revolves around a project created with a bunch of features of React JS from exciting frontend components with Material UI to writing CSS, JSX and making API calls to collect worldwide data related to Corona virus [9]. The major highlights of the project entitled in this paper are no page reloads, Responsiveness, body-parsing, API calls for data collection, JSX (HTML inside JS), sorting algorithms, dynamic data representation, REDUX, data representation in dynamic graphs/pie charts. In this a large number of npm packages like react, react-dom, @material-ui etc along with React JS and CSS library like Material UI has been used in order to code the website such that it results in an attractive, responsive and a beautiful project with a single technology that is JS. The web software entitled in this paper is a complete package of features of React JS integrated with some other frontend technologies like CSS and material UI going through which any user can analyse the data related to corona virus geographically as well as graphically.
\end{abstract}

Manuscript Received November 20, 2020

Kabir Narang, Student, B.Tech, Department of Computer Science \& Engineering, ASET, Amity University Haryana, India (email: kabirnarang39@gmail.com)

Ms. Shivangi Kaushal, Assistant Professor, Department of Computer Science \& Engineering, ASET Amity University Haryana, India

Ms. Ashima Gambhir, Assistant Professor, Department of Computer Science \& Engineering, ASET Amity University Haryana, India
KEYWORDS: Dynamic data representation, Responsiveness, API calls, JSX, Sorting algorithms, NPM packages, Page reloads, JSON, Library, components.

\section{INTRODUCTION}

“COVID19 TRACKER" is a useful application with a bunch of exciting features that it offers to the users from no page reloads to all corona virus related data under a single web page. It covers all scenarios to achieve the requirements of any user searching for worldwide or country wise details regarding corona virus. This application is developed for serving its users to the fullest of it's potential by providing them a platform where they can get the data and information about corona virus not only worldwide but also country wise and in graphical as well as geographical display. Though the application is based more on the frontend development but dynamic data is also given equal preference. The data is collected by asynchronous API calls to disease.sh that provides dynamic data depending on the endpoint used during the call. In simple words disease.sh acts as a server to which we make GET request to provided endpoint and according to endpoint it gives JSON data in response.

\section{RELATED WORK}

In the world of web development React JS as a technology provides a way better approach to represent data. And for any analyser user representation of the data is everything. It is more convenient to user if it is able to gather all information from a single source and that too on just clicks of a button. Data analysis and visualization is a necessary tool for exploring and communicating findings in medical research, especially in epidemiological surveillance [3]. Many government agencies, universities and companies have launched the applications that helps analysing real time situation or virus by providing us updates about number of corona virus cases in our surroundings [2] so that conclusions could be made how to protect us from its widespread. Aarogya Setu application in India and COVID Symptom Study in UK are some of the applications provided by the government of respective countries to their citizens to track about the contacts of people in their surroundings and the current situation of COVID-19 such that the chain of cause could be easily detected and its transmission is reduced to an extent by executing plans made with data in consideration. These are apps that track covid19 and require the high priority GPS and Bluetooth permissions 


\section{Realtime Covid19 Tracker Using React}

to track the spread of infection and also give an idea that how close you are of an infected person and in which zone you spend your time in thus calculating your risks of being infected. When considered in field of research on health care these days corona virus has become one of the most important topic of research and development. Before representing any kind of corona virus data its authenticity is everything and fetching right data requires good knowledge of some JS features. As analysis is the something that helps reducing errors, and making things precise but it does so only when done on correct data. React JS as a frontend library can help writing code in components such that everything that you see on the web is a component that makes code reusable and whenever any change is required somewhere whole code need not to be touched and the part that needs an update can be easily modified. From writing reusable components to a virtual DOM it has features that can help not only in prefect representation of data but also in fast execution of changes on screen. It can easily be integrated with REDUX as a store and firebase as database. React JS is one of the biggest field of development these days and is being used by big MNC'S for UI development. Thus use of this kind of a technology may help creating a wonderful UI that helps user to see covid19 related data on a single web page and that too in a small interaction or clicks and thus boosting the process of analyser's who analyse the data to find perfect solutions to cause. As an alarming situation needs an alarming solution thus using React Js as a technology can make interface fastest and representation of data to fullest using a single page with minimal intervention of user. It covers all scenarios to achieve the requirements of any user searching for worldwide or country wise details regarding corona virus. This application is developed for serving its users to the fullest of it's potential by providing them a platform where they can get the data and information about corona virus not only worldwide but also country wise and in graphical as well as geographical display.

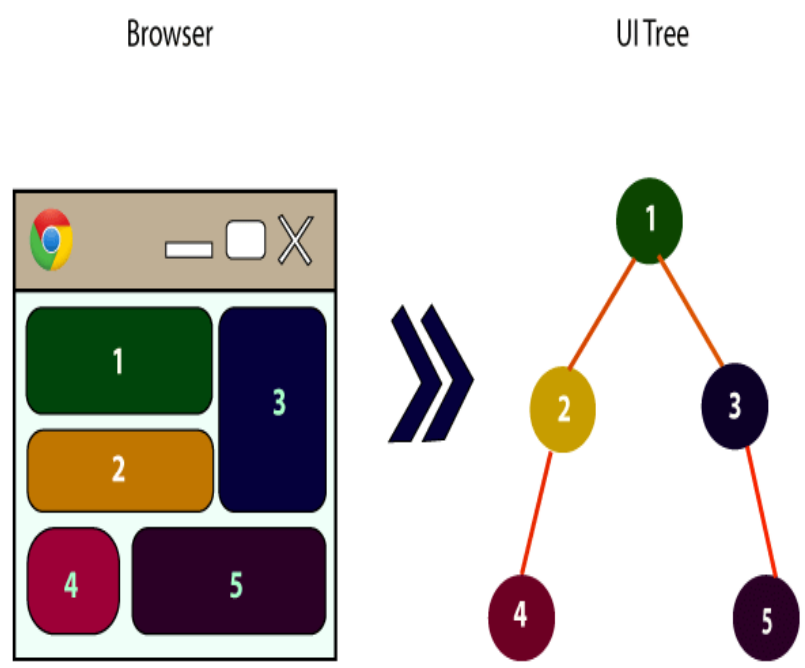

Fig. 1: React JS - A development library that divides DOM in components

Figure 1 above shows that how the whole website is converted in different components and components being on the top of the UI tree being parent components and components being below parent are child components. In React all the properties of parent component can be passed to child component by passing key value pairs to child component when called inside parent component.

\section{METHODOLOGY}

During the development phase of this software certain steps were followed to represent the corona virus related data in the best way possible. Such that precise data is served to a user in different formats including graphical and geographical representation.

Steps followed while development of this project is as follows:

1. Basic structure of the application was created before implementation of any code.

The structure was designed in such a way the whole UI looks as a single web page and there are no too many instructions for the user to reach to a particular data.

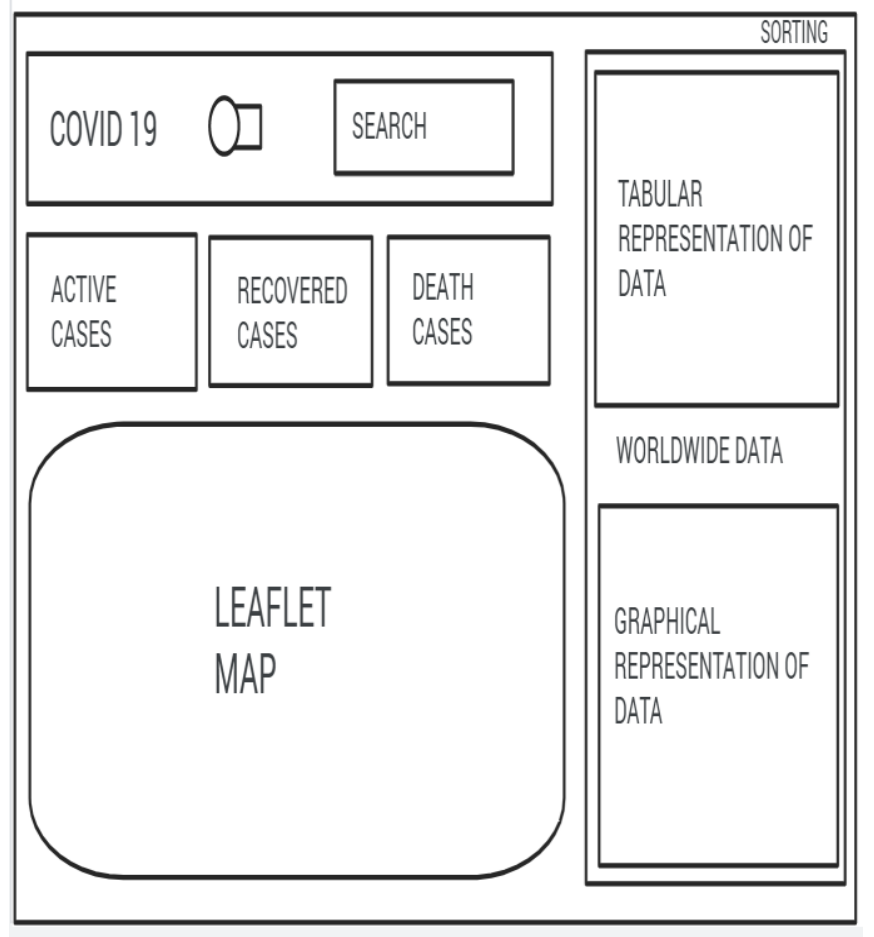

Fig. 2: Basic structure of application

2. Coding phase of the website was initiated and was planned in such a way that when a particular variable changes the component in the DOM associated to it is automatically changed. For example: Map automatically changes as soon as you search.

3. The coding was performed in a hierarchical manner such that parent component was implanted first after that implementation of child components was performed.

For Example: Parent Component: App Child Component: MAP, Search.

4. As the app was coded in components thus making code reusable. 
5. API call were made to disease.sh in order to get data. GET requests were made to endpoints provided by disease.sh.

6. All data fetching calls were made under useEffect() hook provided by React JS that takes two parameters one as an async function describing what to do and second argument as when to run that function.

7. After collection of the data the data was transformed in the required formats as required format for graphs is $\mathrm{X}, \mathrm{Y}$ coordinates.

8. CSS and Material UI are also used as a helping hand to make this website much more attractive.

9. Two modes of web view are also provided for convenience of the user dark mode and light mode.

Forms Workflows

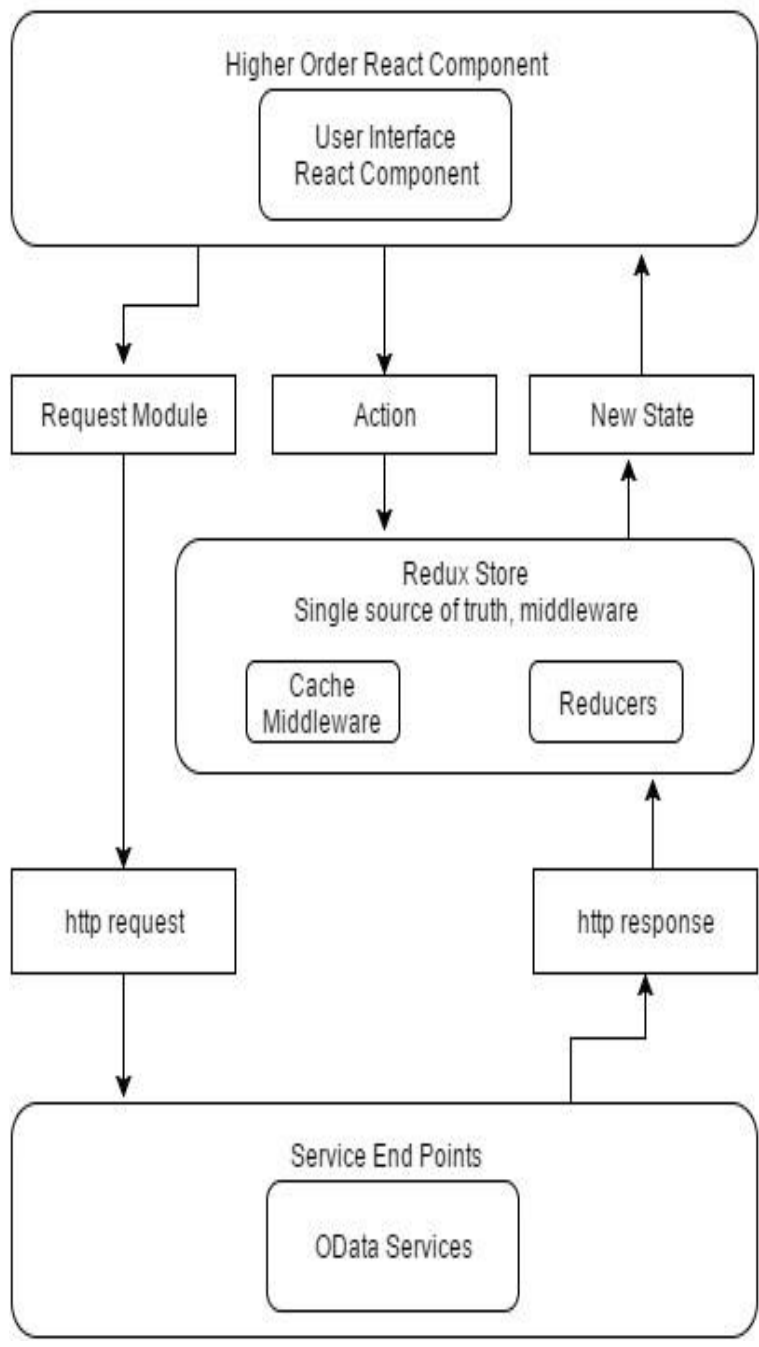

Fig. 3: Architecture of React JS applications

Precise data representation and data modification is the main aim behind a website that serves figures related to any pandemic or healthcare issue [8]. Both of these steps are performed with full attention and using proper techniques of React JS and JS. As serving a web application without any clear motive is of no use so direct and reduced user interaction is maintained throughout the project such that user has to do less but will get more data that too under a single web page.

\section{A. Result}

The resulting web application produced serves as a best example for a software created taken in care of all React JS features and gives user best of UI to use [5]. The representation of data is correctly performed and well managed and colour combinations used in project are well managed in both the modes dark and light. This will let a user coming from any part of the world to either have a look at worldwide data or search according to different countries of choice and not only the data but different forms of data. All these things make this web application easy to use and contribute in providing it responsive and an attractive UI [4].

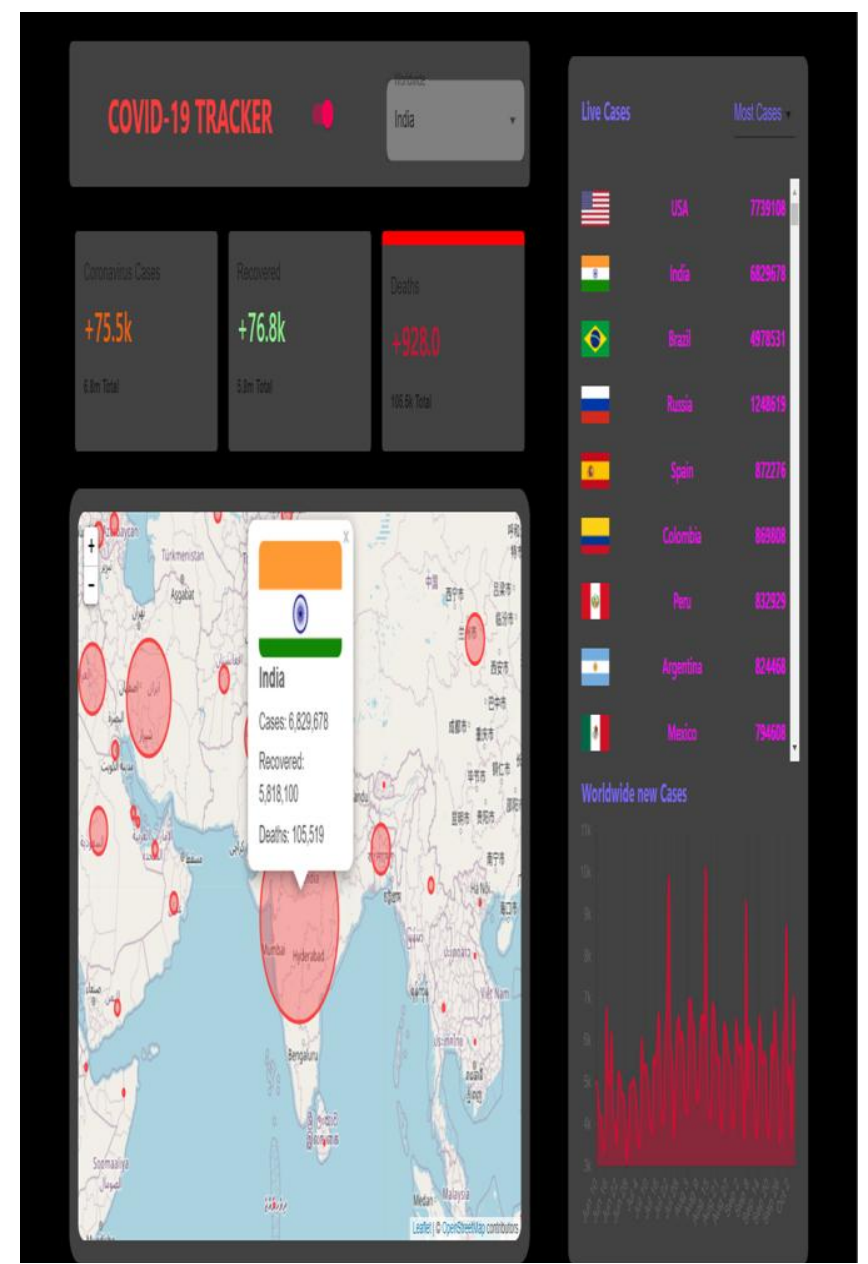

Fig. 4: "COVID 19 TRACKER” Project snapshot in dark mode 


\section{Realtime Covid19 Tracker Using React}
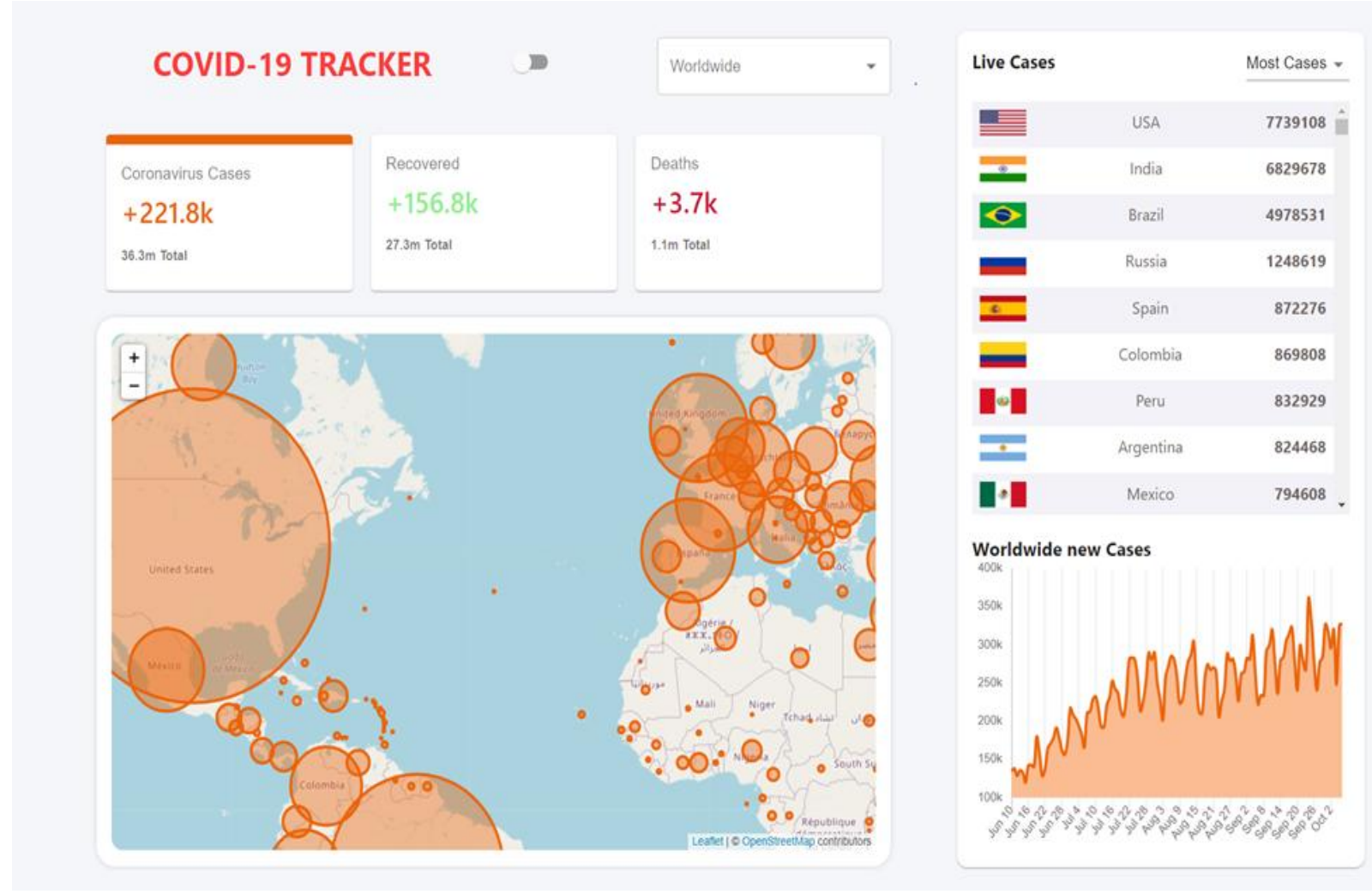

Fig. 5: "COVID 19 TRACKER" Project snapshot in light mode.

As it is very clear from Figure 4 and 5 that whole project is created in components and each component is designed in such a way that a very less user interaction is required but the data represented is more. There is no need for user to search for data from different sources it is just available easily with very less interaction and that too under a single web.

\section{FUTURE SCOPE AND CONCLUSION}

Further implementation of some exciting features like disease prediction on the basis of symptoms and direction of further steps such as finding an appropriate doctor nearby user area would add wonders to this application. These will be helpful in understanding and developing new algorithms for finding doctor's for users according to their area. This is a booming research topic which is still going on for surveillance of large crowds in real time applications. From this application we conclude that how React JS and some basic concepts of frontend development can lead to such an exciting web application with a bunch of features. Though the application is a pretty basic for now but it has a great scope for further implementation of some advanced topics and some more technologies and for now the application is serving its aim to the fullest for which it is developed.

\section{REFERENCES}

[1] Lu R, Zhao X, Li J, Niu P, Yang B, Wu H, et al. Genomic characterisation and epidemiology of 2019 novel coronavirus: implications for virus origins and receptor binding. Lancet 2020. [2]https://www.researchgate.net/publication/341757281_ Case_Study_Fighting_Covid-

19_with_Tech_and_Innovation

[3] Carroll LN, Au AP, Detwiler LT, Fu TC, Painter IS, Abernethy NF. Visualization and analytics tools for infectious disease epidemiology: a systematic review. J Biomed Inform. 2014;51:287-98.

[4] Valls J, Tobias A, Satorra P, Tebe C. COVID19tracker: a shiny app to analise data on SARS-CoV-2 epidemic in Spain. Gac Sanit. 2020;S02139111(20):30085-6. https://doi.org/10.1016/j.gaceta.2020.04.002.

[5] Dong E, Du H, Gardner L. An interactive web-based dashboard to track COVID-19 in real time. Lancet Infect Dis. 2020;20(5):533-4.

[6] Wolkewitz M, Puljak L. Methodological challenges of analysing COVID-19 data during the pandemic. BMC Med Res Methodol. 2020;20(1):81.

[7] Lauer SA, Grantz KH, Bi Q, Jones FK, Zheng Q, Meredith HR, et al. The incubation period of coronavirus disease 2019 (COVID-19) from publicly reported confirmed cases: estimation and application. Ann Intern Med. 2020;172(9):577-82.

[8] Roser M, Ritchie H, Ortiz-Ospina E. Hasell J. Coronavirus Pandemic (COVID-19); 2020. Published online at OurWorldInData.org.Reterivedfrom: https://ourworldindata.org/coronavirus.

[9] https://reactjs.org/docs/ 